\title{
Event-triggered sampling algorithms based on a Lyapunov function
}

\author{
Alexandre Seuret and Christophe Prieur
}

\begin{abstract}
Usually feedback laws are implemented in a periodic fashion on digital hardware. The main reason for using this periodicity in the hardware comes from the difficulties to analyze the stability of aperiodic or asynchronous systems. However it also seems natural to hold the same control input longer if the system behaves in a suitable way or shorter if the system requires an updated input. In this paper, an algorithm is suggested to sample the control input based on the behavior of a Lyapunov-like function. This algorithm is called eventtriggered since the Lyapunov-like function directly depends on the state of the systems.
\end{abstract}

\section{INTRODUCTION}

Over the years, research in control of nonlinear dynamical systems has lead to many different tools to design globally asymptotically stabilizing feedbacks, see e.g. [3], [5], [12], [13]. In the quest for providing more flexible tools for achieving the stabilization and performance tasks, research efforts have focused on analyzing the effect of the switching or on-line adaptation, in particular when implementing the controllers in digital plateforms (see [1] and references therein). This may complicate the analysis of the stability, but also may reduce the cost, i.e. the number of times that the feedback control uses the microprocessors, when closing the loop in digital platforms. Traditionally, the sampling for the controller is chosen periodic, since the analysis of the discrete-time systems has been widely investigated for linear systems [4]. Then several studies deal with the robustness of sampled-data controllers with respect to uncertainties in the sampling instants sequence [7], [8], [20]. These methods ensure the stability of a linear sampled-data system if the sampling period is included in a certain interval. These technics are very relevant but they consider the worst situation. In recent years, an interesting method so-called Event-Based Control suggests to adapt the sampling sequence to some events concerning the state of the system [2], [10], [18], [22].

One application of this kind of algorithms is for the Networked Control Systems (see [11], [23]). Such systems are controlled systems containing several distributed plants which are connected through a communication network. In this situation, the controlled system works in continuoustime whereas the controller provides an discrete-time input which is hold during a sampling period. In this situation, it is relevant to reduce the among of control updates as suggested in [2].

Alexandre Seuret and Christophe Prieur are with Department of Automatic Control, Gipsa-lab, 961 rue de la Houille Blanche, BP 46, 38402 Grenoble Cedex, France, alexandre.seuret@gipsa-lab.grenoble-inp.fr, christophe.prieur@gipsa-lab.grenoble-inp.fr
To tackle this issue, the problem of the design of an eventtriggered algorithm is first rewritten in this paper as the stability study of a system with a mixed continuous/discrete dynamics (also called hybrid system), as considered e.g. [9], [16], [17] in a different context. Using this framework and the Lyapunov theory that is now well known on this kind of nonlinear systems, we compute two new event-triggered algorithms for the implementation of feedback controllers. The first event-triggered algorithm makes a Lyapunov-like function decrease (see Theorem 1 below for a precise statement), whereas the second one allows, in general, to sample less times the control loop to ensure the asymptotic stability of the closed-loop system (see Theorem 2 below). These results are first derived for nonlinear control systems for which it is known a (nonlinear) stabilizing controller. Then the method is particularized to linear control systems and illustrated on (nonlinear and linear) systems borrowed from the literature.

The paper is organized as follows. In Section II some materials on hybrid systems are given, and the problem under consideration in this paper is introduced. In Section III, two event-triggered algorithms are given for nonlinear control systems. Then both main results are applied to the linear case in Section IV. Some numerical simulations illustrate the stability results and the performances of both algorithms in the nonlinear and in the linear cases (see Section V). Section VI contains some concluding remarks and points out some possible open research lines.

Notation. Throughout the article, the sets $\mathbb{N}, \mathbb{R}^{+}, \mathbb{R}^{n}$, $\mathbb{R}^{n \times n}$ and $\mathbb{S}^{n}$ denote respectively the sets of positive integers, positive scalars, $n$-dimensional vectors, $n \times n$ matrices and symmetric matrices of $\mathbb{R}^{n \times n}$. The notation $|\cdot|$ stands for the Euclidean norm. Given a compact set $\mathcal{A}$, the notation $\left.|x|_{\mathcal{A}}=\min \{|x-y|, y \in \mathcal{A}]\right\}$ indicates the distance of the vector $x$ to the set $\mathcal{A}$. The superscript ' $T$ ' stands for matrix transposition. A function $\mu$ is said to be of class $\mathcal{K}_{\infty}$ if it is continuous, zero at zero, increasing and unbounded. The symbols $I$ and 0 represent the identity and the zero matrices of appropriate dimensions.

\section{Problem Formulation}

Consider a continuous-time nonlinear system

$$
\begin{aligned}
\dot{x} & =f(x, u), \\
x\left(t_{0}\right) & =\phi_{0},
\end{aligned}
$$

where $x \in \mathbb{R}^{n}$ and $u \in \mathbb{R}^{m}$ stand respectively for the state variable and the input vector, $\phi_{0} \in \mathbb{R}^{n}$ is the initial state and $f: \mathbb{R}^{n} \times \mathbb{R}^{m} \rightarrow \mathbb{R}^{n}$ is a locally Lipschitz function. 
Assume that the system (1) is globally asymptotically stabilizable, i.e. that there exist a Lyapunov function $V$ and a state feedback control law $\bar{u}$ such that the derivative of the Lyapunov function along the trajectories of the closed-loop system is negative definite. This means that:

Assumption 1: There exist a continuously differentiable function $V: \mathbb{R}^{n} \rightarrow \mathbb{R}$, some functions $\mu_{1}, \mu_{2}$ and $\mu_{3}$ in $\mathcal{K}_{\infty}$ and a continuous controller $u: \mathbb{R}^{n} \rightarrow \mathbb{R}^{m}$ such that $u(0)=0$ and for all $x \in \mathbb{R}^{n}$,

$$
\begin{gathered}
\mu_{1}(|x|) \leq V(x) \leq \mu_{2}(|x|), \\
\nabla V(x) \cdot f(x, u(x)) \leq-\mu_{3}(|x|) .
\end{gathered}
$$

The control law $u$ has been designed in continuoustime. This means that the (continuous)-time derivative of a Lyapunov function is negative definite.

In practice, it is not realistic to implement a control law in continuous-time. As the control input is computed in a digital hardware, only a sampled version of the input is implemented in the actuators. Generally, the sampling is chosen periodic and with a small period so that the sampled signal is very close to the continuous one. However the computation of the control values is not done instantaneously. It requires a minimum sampling period which guarantees that the controller is able to compute the correct data on time. Consequently, the use of a small sampling period requires an efficient processor allowing to compute the control value in short time. An alternative solution is to develop an algorithm which triggers the sampling period with respect to the state of the system, as shown in Figure 1. The contribution of this paper is to let the system decide by itself if an update of the control is needed or not.

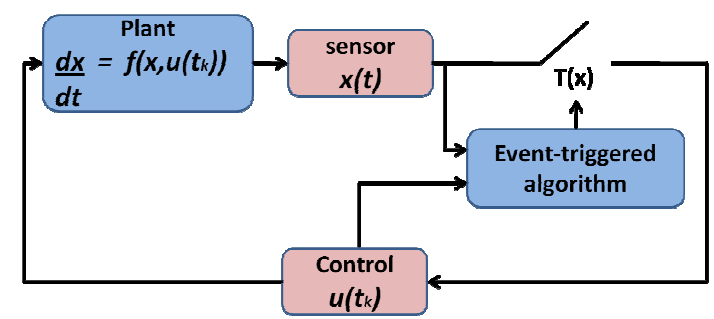

Fig. 1. Control scheme with an event-triggered algorithm.

In order to clarify the notation and the problem under consideration, a hybrid formulation of the sampled-data system is proposed. It was indeed shown in [9], [16], [17] that the sampled-data system can be rewritten as

$$
\begin{aligned}
& \left\{\begin{array}{l}
\dot{x}=f(x, s), \\
\dot{s}=0, \\
\dot{p}=g(x, s, p),
\end{array} \quad \text { if }(x, s, p) \in \mathcal{F},\right. \\
& \left\{\begin{array}{l}
x^{+}=x, \\
s^{+}=u(x), \\
p^{+}=g_{0}(x, s, p),
\end{array} \quad \text { if }(x, s, p) \in \mathcal{J},\right.
\end{aligned}
$$

where $s \in \mathbb{R}^{m}$ represents the held value of the control input, $p \in \mathbb{R}^{q}$ contains additional parameters, $g: \mathbb{R}^{n} \times \mathbb{R}^{m} \times \mathbb{R}^{q} \rightarrow$ $\mathbb{R}^{q}$ and $g_{0}: \mathbb{R}^{n} \times \mathbb{R}^{m} \times \mathbb{R}^{q} \rightarrow \mathbb{R}^{q}$ are two continuous functions of appropriate dimensions, and $\mathcal{F}$ and $\mathcal{J}$ are two subsets of $\mathbb{R}^{n} \times \mathbb{R}^{m} \times \mathbb{R}^{q}$. These sets are respectively called flow set and jump set and are degrees of freedom of the event-triggered algorithm. The variable $s$ represents the held value of $u(x)$, which is the control value that is implemented over the sampling interval.

We recall some basic ingredients on hybrid system theory, and on the notion of solutions of (2) (see [9], [16]). Due to mixed discrete/continuous dynamics, a solution of (2) will be defined on a mixed discrete/continuous time domain. More precisely, a set $E$ is a hybrid time domain if for all $(T, J) \in$ $E, E \cap([0, T] \times\{0,1, \ldots J\})$ is a compact hybrid time domain, i.e. it can be written as

$$
\bigcup_{j=0}^{J-1}\left(\left[t_{j}, t_{j+1}\right], j\right),
$$

for some finite sequence of times $0=t_{0} \leq t_{1} \ldots \leq t_{J}$. A solution $x$ to (2) consists of a hybrid time domain dom and functions $x: \operatorname{dom} \rightarrow \mathbb{R}^{n}, s: \operatorname{dom} \rightarrow \mathbb{R}^{m}$, and $p: \operatorname{dom} \rightarrow \mathbb{R}$ such that $(x, s, p)(t, j)$ is absolutely continuous in $t$ for a fixed $j$ and $(t, j) \in$ dom satisfying

(S1) for all $j \in \mathbb{N}$ and almost all $t$ such that $(t, j) \in$ dom,

$$
\begin{aligned}
x(t, j) \in F, \dot{x}(t, j) & =f(x(t, j), s(t, j)), \\
\dot{s}(t, j) & =0, \\
\dot{p}(t, j) & =g(x(t, j), s(t, j), p(t, j)),
\end{aligned}
$$

(S2) for all $(t, j) \in$ dom such that $(t, j+1) \in$ dom,

$$
\begin{aligned}
x(t, j) \in J, x(t, j+1) & =x(t, j), \\
s(t, j+1) & =u(x(t, j)), \\
p(t, j+1) & =g(x(t, j), s(t, j), p(t, j)) .
\end{aligned}
$$

Then, the state solution $x$ is parameterized by $(t, j)$ where $t$ is the ordinary time and $j$ is an independent variable that corresponds to the number of jumps of the solution. When the state $x(t, j)$ belongs to the intersection of the flow set and of the jump set, then the solution can either flow or jump. This parameterization may be omitted when there is no ambiguity.

A solution $x$ to (2) is said to be complete if dom is unbounded, Zeno if it is complete but the projection of dom onto $\mathbb{R}_{\geq 0}$ is bounded, and maximal if there does not exist an other solution $\tilde{x}$ of (2) such that $x$ is a truncation of $\tilde{x}$ to some proper subset of dom. Hereafter, only maximal solutions will be considered. For more details about this hybrid systems framework, we refer the reader to [9], [16]. The following definition describes the requirements to prove the global asymptotic stability of the solutions of (2).

Definition 2.1: Given a compact subset $\mathcal{A}$ of $\mathbb{R}^{n} \times \mathbb{R}^{m} \times$ $\mathbb{R}^{q}$ the hybrid system (2) is said to be

- stable to $\mathcal{A}$ : if for each $\varepsilon>0$ there exists $\delta>0$ such that each solution $(x, s, p)$ to (2) with $|(x(0,0), s(0,0), p(0,0))|_{\mathcal{A}} \leq \delta$ satisfies $|(x(t, j), s(t, j), p(t, j))|_{\mathcal{A}} \leq \varepsilon$ for all $(t, j) \in \mathrm{dom} ;$

- attractive to $\mathcal{A}$ : if every solution $x$ to (2) is complete and satisfies $\lim _{t+j \rightarrow \infty}|(x(t, j), s(t, j), p(t, j))|_{\mathcal{A}}=0$; 
- globally asymptotically stable to $\mathcal{A}$ : if it is both stable and attractive to $\mathcal{A}$.

Given an initial condition $\left(\phi_{0}, s_{0}, p_{0}\right)$ in $\mathbb{R}^{n} \times \mathbb{R}^{m} \times \mathbb{R}$, and a solution $(x, s, p)$ of (2) defined on a hybrid time domain dom, the set of the sampling time instants, when the control input is updated, (plus 0) is denoted $\mathcal{T}$ and is $\left\{t_{j}\right\}$, where $t_{j}$ is such dom $=\cup_{j \in J}\left(\left[t_{j}, t_{j+1}\right], j\right)$. Among other results, we will prove in this paper some properties on the set $\mathcal{T}$ depending on the choice of the event-triggered algorithm. In particular, in our hybrid systems framework, $\mathcal{T}$ is at most countable.

In this paper, different sets $\mathcal{F}$ and $\mathcal{J}$ are defined, and thus different event-triggered algorithms are considered. Let, as a first example, the particular case where $p=\tau \in \mathbb{R}$ and such that the dynamics of the system is rewritten, for any $T>0$ as

$$
\begin{aligned}
& \left\{\begin{array}{l}
\dot{x}=f(x, s), \\
\dot{s}=0, \\
\dot{\tau}=1,
\end{array} \quad \text { if }(x, s, \tau) \in \mathcal{F}_{T},\right. \\
& \left\{\begin{array}{l}
x^{+}=x, \\
s^{+}=u(x), \\
\tau^{+}=0,
\end{array}\right.
\end{aligned}
$$

where $\mathcal{F}_{T}$ and $\mathcal{J}_{T}$ are the following subsets of $\mathbb{R}^{n} \times \mathbb{R}^{m} \times$ $[0, T]$ :

$$
\begin{aligned}
& \mathcal{F}_{T}=\{x, s, \tau, \text { such that } \tau \leq T\}, \\
& \mathcal{J}_{T}=\{x, s, \tau, \text { such that } \tau \geq T\} .
\end{aligned}
$$

As shown in [9], the hybrid model expresses the case of periodic sampling. In this simple algorithm, after each jump, the solution is either at the equilibrium or has to flow. It avoids the existence of Zeno solutions, and also it reduces the complexity when implementing the eventtriggered algorithm. Of course, in general, the system (3) is not globally asymptotically stable since the update of the control law does not depend on the system position but is done periodically. This motivates us to consider the following problem:

Problem 1: Define appropriate sets $\mathcal{F}$ and $\mathcal{J}$ and $d y$ namics of the variable $p$ such that, after each jump of the solutions of (2), the solutions have to flow, and such that (2) is globally asymptotically stable.

\section{EVENT-TRIGGERED ALGORITHM FOR NONLINEAR SYSTEMS}

\section{A. A continuous-time decreasing function}

In this section, the objective is to define flow and jump sets which are based on the decay of the function in continuoustime.

Theorem 1: Under Assumption 1, consider a given function $\mu$ of class $\mathcal{K}_{\infty}$ such that $\mu(r)<\mu_{3}(r)$, for all $r>0$. Consider the flow and jump sets given by

$$
\begin{aligned}
& \mathcal{F}_{1}=\{(x, s), \nabla V(x) \cdot f(x, s) \leq-\mu(|x|)\}, \\
& \mathcal{J}_{1}=\{(x, s), \nabla V(x) . f(x, s) \geq-\mu(|x|)\},
\end{aligned}
$$

and the associated event-triggered algorithm. Then the system (2) with $\mathcal{F}=\mathcal{F}_{1}$ and $\mathcal{J}=\mathcal{J}_{1}$ (and without state $p$ ) is globally asymptotically stable to the origin. Moreover, after each jump of the solutions of this hybrid system, either the state is the origin or the solution has to flow.

Proof. The proof of Theorem 1 is based on the decreasing property of the function $V$ given by Assumption 1, along the solutions of (2), with $\mathcal{F}$ and $\mathcal{J}$ given by (5). See [17] for analogous ideas for a different problem.

Given a switching time instant $t_{0} \in \mathcal{T}$, denoting (with a slight abuse of notation) $x\left(t_{0}^{+}\right)$the state after the jump (and similarly for the other variables), using Assumption 1, it yields

$$
\begin{aligned}
\nabla V\left(x\left(t_{0}^{+}\right)\right) \cdot f\left(x\left(t_{0}^{+}\right), s\left(t_{0}^{+}\right)\right) & =\nabla V(x) \cdot f\left(x\left(t_{0}^{+}\right), u\left(x\left(t_{0}^{+}\right)\right)\right) \\
& \leq-\mu_{3}\left(\left|x\left(t_{0}^{+}\right)\right|\right) \\
& \leq-\mu\left(\left|x\left(t_{0}^{+}\right)\right|\right)-\varepsilon\left(\left|x\left(t_{0}^{+}\right)\right|\right),
\end{aligned}
$$

where $\varepsilon\left(\left|x\left(t_{0}^{+}\right)\right|\right)=\mu_{3}\left(\left|x\left(t_{0}^{+}\right)\right|\right)-\mu\left(\left|x\left(t_{0}^{+}\right)\right|\right)$is non-negative and equals 0 only if $x\left(t_{0}^{+}\right)$is vanishing. Thus, after a jump, two cases may occur:

1) either the state $x$ is at the origin (and the same for the other components), and then the solution remains at the origin;

2) or $\left(x\left(t_{0}^{+}\right), s\left(t_{0}^{+}\right)\right)$belongs to $\mathcal{F}_{1}$ and is different to the origin.

Consider now $(x, s)$ in $\mathcal{F}_{1} \backslash\{0\}$. Then we get

$$
\begin{aligned}
\nabla V(x) \cdot f(x, s)= & \nabla V(x) \cdot(f(x, s)-f(x, u(x))) \\
& +\nabla V(x) \cdot f(x, u(x)),
\end{aligned}
$$

and using Assumption 1, we obtain

$$
\begin{aligned}
\nabla V(x) . f(x, s) \leq & -\mu_{3}(|x|) \\
& +\nabla V(x) \cdot(f(x, s)-f(x, u(x))) .
\end{aligned}
$$

Then, the solution $(x, s)$ of system (2) with $\mathcal{F}=\mathcal{F}_{1}$ and $\mathcal{J}=\mathcal{J}_{1}$ stays in $\mathcal{F}_{1}$ until a state $x=x^{*}$ (if such a state does exist) defined by

$$
\nabla V\left(x^{*}\right) \cdot\left(f\left(x^{*}, s\right)-f\left(x^{*}, u\left(x^{*}\right)\right)\right)=\mu_{3}\left(\left|x^{*}\right|\right)-\mu\left(\left|x^{*}\right|\right) .
$$

Two subcases may occur.

2.a) If there exists such $x^{*}$, then the couple $\left(x^{*}, s\right)$ belongs to $\mathcal{J}_{1}$, and by definition of $s^{+},\left(x^{*+}, s^{+}\right)$belongs to $\mathcal{F}_{1}$.

2.b) If there does not exist such $x^{*}$, then the solution of the system (2) stays in $\mathcal{F}_{1}$.

For both cases, the derivative of $V$ is negative while $(x, s)$ is in $\mathcal{F}_{1}$ and $V$ is constant while $(x, s)$ is in $\mathcal{J}_{1}$. This implies that the system (2) with $\mathcal{F}=\mathcal{F}_{1}$ and $\mathcal{J}=\mathcal{J}_{1}$ is stable.

To prove the attractivity of the system (2) with $\mathcal{F}=\mathcal{F}_{1}$ and $\mathcal{J}=\mathcal{J}_{1}$, let us apply the LaSalle invariance property for hybrid systems. Let us consider a solution of this hybrid system which is included in a level set of the function $V$. Let us show that this solution should be equal to 0 .

The solution cannot jump (since after a jump, the solution has to flow, and thus the value of $V$ has to decrease). Given a solution flowing for all time, due to Assumption 1, the state $x$ cannot stay at the level set of $V$. Thus the solution has to be constant and equal to the origin. Therefore, by [9, Theorem 23, page 64], the system (2) with $\mathcal{F}=\mathcal{F}_{1}$ and $\mathcal{J}=\mathcal{J}_{1}$ is globally asymptotically stable. This concludes the proof of Theorem 1 . 
Remark 1: A main improvement of the proposed method compared for example to [1], is that no Input-to-State Stability (ISS) assumption for system (1) is needed. More precisely the method that is suggested in [1] requires the existence of functions $\alpha$ and $\gamma$ of class $\mathcal{K}_{\infty}$, such that, for all $x$ in $\mathbb{R}^{n}$,

$$
\nabla V(x) \cdot f(x, s) \leq-\alpha(|x|)+\gamma(|u(x)-s|)
$$

Then the event-triggered algorithm is defined by a condition on the error between the control value $s$ and the controller $u$ that is:

$$
|u(x)-s| \leq \gamma^{-1}(\sigma \alpha(|x|))
$$

where $\sigma$ in $(0,1)$ is a tuning parameter. In the present article, instead of an ISS assumption, only the global asymptotic stability is needed. As remarked in [21] e.g., Assumption 1 is weaker than the ISS property, and it is sufficient to define the event-triggered algorithm by the value of the derivative of the Lyapunov function along the trajectories of the system.

Remark 2: Another important issue concerns the possibility that the solution of the system for a given initial condition, never reaches the set $\mathcal{J}_{1}$. In such situation, it simply means that the system is already asymptotically stable without any control (or with a constant control value) and the control law does not need to be updated. This situation is not taken into account in the method proposed in [1]. 0

Remark 3: On the other side, the main drawback of the method proposed in this article is the real time computation of the derivative of the Lyapunov function $V$.

Note that picking $\mu=0$ in Theorem 1 gives a partial result and allows to design an event-triggered algorithm such that the closed-loop system is globally stable. More precisely we have

Proposition 1: Under Assumption 1, consider the flow and jump sets given by

$$
\begin{aligned}
\mathcal{F}_{1}^{\prime} & =\{(x, s), \nabla V(x) . f(x, s) \leq 0\}, \\
\mathcal{J}_{1}^{\prime} & =\{(x, s), \nabla V(x) . f(x, s) \geq 0\},
\end{aligned}
$$

and the associated event-triggered algorithm. Then the systems (2) with $\mathcal{F}=\mathcal{F}_{1}^{\prime}$ and $\mathcal{J}=\mathcal{J}_{1}^{\prime}$ is globally stable. Moreover, after each jump of the solutions of this hybrid system, either the state is the origin or the solution has to flow.

Proof. The proof follows the lines of Theorem 1. More precisely, we may check that by selecting $\mathcal{F}=\mathcal{F}_{1}^{\prime}$ and $\mathcal{J}=\mathcal{J}_{1}^{\prime}$, and by using Assumption 1, the derivative of the Lyapunov function $V$ is negative while the state of the solution $(x, s)$ of (2) is in $\mathcal{F}_{1}$ and is constant $(x, s)$ is in $\mathcal{J}_{1}$. This implies, with [9, Theorem 23, page 64], that the system (2) with $\mathcal{F}=\mathcal{F}_{1}$ and $\mathcal{J}=\mathcal{J}_{1}$ is stable.

Finally, using Assumption 1 again, we note that, given a solution of (2) with $\mathcal{F}=\mathcal{F}_{1}^{\prime}$ and $\mathcal{J}=\mathcal{J}_{1}^{\prime}$, after each jump (if such a jump does exist), either the state is the origin or the solution has to flow.

\section{B. A discrete-time decreasing function}

In this section, the objective is to define flow and jump sets which are based both on the decay of the function in continuous-time and in discrete-time. A second eventtriggered algorithm is given by the following system

$$
\left\{\begin{array}{l}
\dot{x}=f(x, s), \\
\dot{s}=0, \\
\dot{v}=0, \\
\dot{\tau}=1, \\
x^{+}=x, \\
s^{+}=u(x), \\
v^{+}=\lambda V(x), \\
\tau^{+}=0,
\end{array}\right.
$$

where $v$ is an additional state in $\mathbb{R}, \lambda \in(0,1)$. This is system (2) with a particular choice of the function $g$ and $g_{0}$. A design of an event-triggered algorithm for the control system (1) is given by the following:

Theorem 2: Under Assumption 1, consider a sufficiently large $T>0$ and a function $\mu$ of class $\mathcal{K}_{\infty}$ such that $\mu(r)<$ $\mu_{3}(r)$, for all $r>0$. Consider the flow and jump sets given by

$$
\mathcal{F}_{2}=\left(\overline{\mathcal{F}}_{1} \cup \overline{\mathcal{F}}_{2}\right) \bigcap \mathcal{F}_{T}, \quad \mathcal{J}_{2}=\left(\overline{\mathcal{J}}_{1} \bigcap \overline{\mathcal{J}}_{2}\right) \cup \mathcal{J}_{T}
$$

where

$$
\begin{array}{ll}
\overline{\mathcal{F}}_{1}=\{(x, s, v, \tau), & \left.(x, s) \in \mathcal{F}_{1}\right\}, \\
\overline{\mathcal{J}}_{1}=\{(x, s, v, \tau), & \left.(x, s) \in \mathcal{J}_{1}\right\}, \\
\overline{\mathcal{F}}_{2}=\{(x, s, v, \tau), & V(x) \leq v\}, \\
\overline{\mathcal{J}}_{2}=\{(x, s, v, \tau), & V(x) \geq v\},
\end{array}
$$

and where $\mathcal{F}_{T}$ and $\mathcal{J}_{T}$ are defined by (4). Then the system (6) with the event-triggered algorithm derived from $\mathcal{F}=\mathcal{F}_{2}$ and $\mathcal{J}=\mathcal{J}_{2}$ is globally asymptotically stable to the set $\{0\} \times\{0\} \times\{0\} \times[0, T]$. Moreover, after each jump of the solutions of this hybrid system, either the state is the origin or the solution has to flow.

Proof. The introduction of the sets $\mathcal{F}_{T}$ and $\mathcal{J}_{T}$ ensures that there are at most $T$ units of continuous time between two jumps. Moreover, for each solution of the system (6) with the event-triggered algorithm derived from $\mathcal{F}=\mathcal{F}_{2}$ and $\mathcal{J}=\mathcal{J}_{2}$, due to the discrete dynamics equation, after each jump, the solution has to flow.

Note that since the flow set $\mathcal{F}_{2}$ and the jump sets $\mathcal{J}_{2}$ are closed, and since the dynamics of the system (6) are continuous, then, with [9, Thereom S3], each maximal solution is either complete (i.e. with a unbounded time domain) or blows up in finite time.

Let us show, by stating a contradiction, that the solution cannot blow up in finite time. To do that let us assume that it is given a solution of (6) with the event-triggered algorithm derived from $\mathcal{F}=\mathcal{F}_{2}$ and $\mathcal{J}=\mathcal{J}_{2}$ such that the solution blows up in (hybrid) finite time. Inspecting the variables $x, s, v$ and $\tau$, if the solution is blowing up in finite continuous time, then the $x$-variable has to blow up in finite continuous time (the other components are bounded). However, by definition of $\mathcal{F}_{1}$, the $V$ function cannot blow up. Therefore, if there exists a blow up in (hybrid) finite time, 
then it should blow up in the discrete time direction. Now let us remark that between two jumps, due the dynamics of the $v$ variable, the $V$ function cannot increase. It gives a contradiction with the blow up of the $x$-variable in discrete time.

Let us state now that the system (6) with the eventtriggered algorithm derived from $\mathcal{F}=\mathcal{F}_{2}$ and $\mathcal{J}=\mathcal{J}_{2}$ is globally attractive to $\{0\} \times\{0\} \times\{0\} \times[0, T]$. To do that, let us consider a solution $(x, s, v, \tau)$ of system (6) with the eventtriggered algorithm derived from $\mathcal{F}=\mathcal{F}_{2}$ and $\mathcal{J}=\mathcal{J}_{2}$. Moreover let us denote $\mathcal{T}$ the set of (continuous) sampling time instants, when the control input is updated (plus 0 ).

The set $\mathcal{T}=\left\{t_{k}\right\}$ is infinite since the solution is complete, and, between two jumps, there is at most $T$ units of time. Let us denoting $V_{k}$, and $x_{k}$ the value of the $V$ function and of the $x$-variable, when there is a jump. Due to Assumption 1 and the inequality $\mu(r)<\mu_{3}(r)$ for all $r>0$, we have $V_{k}<$ $V_{k-1}$, for all $k$ in $\mathbb{N}$, and thus $x_{k}$ converges to zero when $k$ tends to the infinity. Now, by the continuous differentiability of the functions in the right-hand side of the system (6), we have the convergence of the $s$ - and $v$-components of the solution at the switching time instants. Thus, by definition of the set $\overline{\mathcal{F}}_{2}$, and since the $v$-variable converges to zero at the switching time instants, the $x$-variable tends also to zero when the hybrid time tends to the infinity. By the continuous differentiability of the functions in the right-hand side of the system (6), it concludes the proof of the attractivity of the system (6) with the event-triggered algorithm derived from $\mathcal{F}=\mathcal{F}_{2}$ and $\mathcal{J}=\mathcal{J}_{2}$ to the set $\{0\} \times\{0\} \times\{0\} \times[0, T]$.

The local stability of the system (6) with the eventtriggered algorithm derived from $\mathcal{F}=\mathcal{F}_{2}$ and $\mathcal{J}=\mathcal{J}_{2}$ follows from the fact that between two switches the $V$ function does not increase and that the $V$ function is bounded by the $v$-variable between two switches.

This concludes the proof of Theorem 2 .

Remark 4: Compared to the event-driven algorithm which is described in Theorem 1, the above one could lead to a larger sampling period since it allows the function $V$ to be locally increasing. This is the main improvement of the second algorithm with respect to the first one (as remarked on the numerical simulations of Section $\mathrm{V}$ below). On this other hand the performance and the convergence rate of the system might be worst than using Theorem 1 since the second algorithm allows an increase of the function $V$. Here appears a tradeoff between the minimization of the number of updates and the performance of the closed-loop system. We will come back to this issue in the conclusion section. $\circ$

Remark 5: An important issue has to do with the choice of the parameter $\lambda$. If $\lambda$ is chosen too small, it may happen that the solutions of the system never reaches $\overline{\mathcal{F}}_{2}$. This means that the event-driven algorithm defined by the sets $\mathcal{F}_{2}$ and $\mathcal{J}_{2}$ is exactly the same than the one defined in Theorem 1. Another interest of the event-driven algorithm which is proposed in Theorem 2 is that there is no need to ensure the existence of a particular value of $\lambda$ such that the sets $\mathcal{F}_{2}$ is not empty.

\section{AppliCATION TO LINEAR SYSTEMS}

\section{A. A continuous-time decreasing function}

Consider now a linear system of the form

$$
\dot{x}=A x+B u,
$$

where $x \in \mathbb{R}^{n}$ and $u \in \mathbb{R}^{m}$ stand for the state variable and the input vector. The matrices $A$ and $B$ are constant and known and of appropriate dimensions. Let us assume that the pair $(A, B)$ is controllable. Then the proposed control law for this system is $u=K x$ where $K$ in $\mathbb{R}^{m \times n}$ is such that the matrix $A+B K$ is Hurwitz. There also exist a positive scalar $\alpha$ and a symmetric positive definite matrix $P$ so that

$$
P(A+B K)+(A+B K)^{T} P<-2 \alpha P .
$$

Thus Assumption 1 holds with $V(x)=x^{T} P x$ and $u(x)=$ $K x$ for all $x \in \mathbb{R}^{n}$. Rewriting the closed loop system in a hybrid framework, we get:

$$
\begin{cases}\dot{x}=A x+B s, & \text { if }(x, s) \in \mathcal{F}, \\ \dot{s}=0, & \text { if }(x, s) \in \mathcal{J} . \\ x^{+}=x, & \end{cases}
$$

By noting that

$$
\nabla V(x) f(x, s)=\left[\begin{array}{l}
x \\
s
\end{array}\right] \Pi_{1}\left[\begin{array}{l}
x \\
s
\end{array}\right]
$$

where

$$
\Pi_{1}=\left[\begin{array}{cc}
P A+A^{T} P+2 \bar{\alpha} P & P B K \\
(B K)^{T} P & 0
\end{array}\right], \bar{\alpha} \in(0, \alpha),
$$

the following result follows readily from Theorem 1:

Proposition 2: Assume there exist a symmetric positive definite matrix $P$ in $\mathbb{R}^{n \times n}$, a matrix $K$ in $\mathbb{R}^{n \times m}$ and a positive scalar $\alpha$ satisfying (8). Consider $\bar{\alpha} \in(0, \alpha)$ and the flow and jump sets defined by

$$
\left.\begin{array}{ll}
\mathcal{F}_{1 L}=\{(x, s), & {\left[\begin{array}{c}
x \\
s
\end{array}\right]^{T} \Pi_{1}\left[\begin{array}{l}
x \\
s
\end{array}\right] \leq 0}
\end{array}\right\},
$$

Then the system (9) with the event-triggered algorithm derived from $\mathcal{F}=\mathcal{F}_{1 L}$ and $\mathcal{J}=\mathcal{J}_{1 L}$ is globally asymptotically stable to the origin. Moreover, after each jump of the solutions of this hybrid system, either the state is the origin or the solution has to flow.

\section{B. A discrete-time decreasing function}

Following the idea proposed in Theorem 2, we will consider the hybrid system defined by

$$
\begin{aligned}
& \left\{\begin{array}{l}
\dot{x}=A x+B s, \\
\dot{s}=0, \\
\dot{v}=0, \\
\dot{\tau}=1,
\end{array} \quad \text { if }(x, s, v, \tau) \in \mathcal{F},\right. \\
& \left\{\begin{array}{l}
x^{+}=x, \\
s^{+}=K x, \\
v^{+}=\lambda V(x), \\
\tau^{+}=0,
\end{array}\right.
\end{aligned}
$$


By applying Theorem 2, we obtain the following:

Proposition 3: Assume there exist a symmetric positive definite matrix $P$ in $\mathbb{R}^{n \times n}$, a matrix $K$ in $\mathbb{R}^{n \times m}$ and a positive scalar $\alpha$ satisfying (8). Consider $\bar{\alpha} \in(0, \alpha)$ and the flow and jump sets given by

$$
\mathcal{F}_{2 L}=\left(\overline{\mathcal{F}}_{1 L} \bigcup \overline{\mathcal{F}}_{2 L}\right) \bigcap \mathcal{F}_{T}, \quad \mathcal{J}_{2 L}=\left(\overline{\mathcal{J}}_{1 L} \bigcap \overline{\mathcal{F}}_{2 L}\right) \bigcup \mathcal{F}_{T},
$$

where

$$
\begin{array}{ll}
\overline{\mathcal{F}}_{1 L}=\{(x, s, v, \tau), & \left.(x, s) \in \mathcal{F}_{1 L}\right\}, \\
\overline{\mathcal{J}}_{1 L}=\{(x, s, v, \tau), & \left.(x, s) \in \mathcal{J}_{1 L}\right\}, \\
\overline{\mathcal{F}}_{2 L}=\{(x, s, v, \tau), & \left.x^{T} P x \leq v\right\}, \\
\overline{\mathcal{J}}_{2 L}=\{(x, s, v, \tau), & \left.x^{T} P x \geq v\right\} .
\end{array}
$$

Then the system (10) with the event-triggered algorithm derived from $\mathcal{F}=\mathcal{F}_{2 L}$ and $\mathcal{J}=\mathcal{J}_{2 L}$ is globally asymptotically stable to the set $\{0\} \times\{0\} \times\{0\} \times[0, T]$. Moreover, after each jump of the solutions of this hybrid system, either the state is the origin or the solution has to flow.

\section{Comments on the linear case}

The event-triggered algorithms which are exposed above do not provide any information of the duration while a control law is held. In the sequel, a complementary analysis is provided for the case of linear systems to give an upperbound and a lower bound of the holding times.

Let $\chi \in \mathbb{R}^{n}$ be the value of $x$-component in the system (10) with the event-triggered algorithm derived from $\mathcal{F}=$ $\mathcal{F}_{i L}$ and $\mathcal{J}=\mathcal{J}_{i L}, i=1,2$, at an instant when the system is jumping, i.e. $\chi=x\left(t_{j}\right)$ for some $t_{j} \in \mathcal{T}$. In the case of linear sampled-data systems, the relations between $\chi, x, s$, $v$ and $\tau$ are given by

$$
\begin{aligned}
& x\left(t_{k}+\tau\right)=\Gamma(\tau) \chi, s\left(t_{k}+\tau\right)=K \chi, \\
& v\left(t_{k}+\tau\right)=\lambda \chi^{T} P \chi,
\end{aligned}
$$

where $\Gamma(\tau)=e^{A \tau}+\int_{0}^{\tau} e^{A(\theta-\tau)} d \theta B K$. For the sake of simplicity, we will denote

$$
\begin{aligned}
& X_{1}(\chi, \tau)=(\Gamma(\tau) \chi, K \chi), \quad \text { for algorithm } 1 \text { and } \\
& X_{2}(\chi, \tau)=(\Gamma(\tau) \chi, K \chi, v, \tau), \quad \text { for algorithm } 2
\end{aligned}
$$

for any given $\chi \in \mathbb{R}^{n}$ and $T \in \mathbb{R}^{+}$. Based on these linking relations, bounds on the sampling periods can be provided. This is stated in the sequel.

Proposition 4: Consider the linear system (10) with the event-triggered algorithm derived from $\mathcal{F}=\mathcal{F}_{i L}$ and $\mathcal{J}=$ $\mathcal{J}_{i L}, i=1,2$. Then, the difference between two successive sampling instants is included in the interval $\left[T_{\min }^{i}, T_{\max }^{i}\right]$ defined as follows

$$
\begin{aligned}
& T_{\text {max }}^{i}=\max _{\rho \in \mathbb{R}^{n},|\rho|=1}\left\{\max _{X_{i}(\rho, \tau) \in \mathcal{F}_{i L}} \tau\right\} \\
& T_{\text {min }}^{i}=\min _{\rho \in \mathbb{R}^{n},|\rho|=1}\left\{\max _{X_{i}(\rho, \tau) \in \mathcal{F}_{i L}} \tau\right\}
\end{aligned}
$$

Proof. Consider any state $\chi$ in $\mathbb{R}^{n}$ for a solution of system (10) with the event-triggered algorithm derived from $\mathcal{F}=$ $\mathcal{F}_{i L}$ and $\mathcal{J}=\mathcal{J}_{i L}$ when a jump is occurring. By simple computations, it is clear that if, for any $\tau>0, X_{i}(\chi, \tau)$ belongs to $\mathcal{F}_{i L}$ then $X_{i}(\chi /|\chi|, \tau)$ also belongs to $\mathcal{F}_{i L}$. Then, from the definition of $T_{\min }^{i}$ and $T_{\text {max }}^{i}$, the next update will happen between these two bounds.

\section{NUMERICAL EXAMPLES}

\section{A. Nonlinear example}

Consider the following nonlinear system borrowed from [1], [3]:

$$
\left\{\begin{array}{l}
\dot{x}_{1}=u_{1}, \\
\dot{x}_{2}=u_{2}, \\
\dot{x}_{3}=x_{1} x_{2},
\end{array}\right.
$$

where $\left(x_{1}, x_{2}, x_{3}\right)$ and $\left(u_{1}, u_{2}\right)$ stand respectively for the state and for the control. A stabilizing controller is computed in [3]. It is given by, for all $\left(x_{1}, x_{2}, x_{3}\right)$ in $\mathbb{R}^{3}$,

$$
\left\{\begin{array}{l}
u_{1}\left(x_{1}, x_{2}\right)=-x_{1} x_{2}-2 x_{2} x_{3}-x_{1}-x_{3}, \\
u_{2}\left(x_{1}, x_{2}\right)=2 x_{1} x_{2} x_{3}+3 x_{3}^{2}-x_{2} .
\end{array}\right.
$$

A Lyapunov function for this system is computed in the same reference. It is defined by, for all $\left(x_{1}, x_{2}, x_{3}\right)$ in $\mathbb{R}^{3}$,

$$
V(x)=\left(x_{1}+x_{3}\right)^{2} / 2+\left(x_{2}-x_{3}^{2}\right)^{2} / 2+x_{3}^{2} .
$$

Thus Assumption 1 holds. The simulation results are shown in Figure 2 where three different numerical simulations are done: 1) the closed-loop system in continuous-time, 2) and the event-triggered algorithms which are presented in Theorem 1,3 ) and with Theorem 2 . The following parameters have been selected $\mu(x)=10^{-3}|x|^{4}, \lambda=0.2$. The initial conditions are $x_{1}(0)=-10, x_{2}(0)=-5$ and $x_{3}(0)=5$. The simulation results are shown in Figure 2. The figure contains the state, the function $V$, the variable $\tau$ defined in (3), representing the sampling periods and the input variable. The first and the second algorithms require respectively 20 and 14 sampling instants over a simulation time of $20 \mathrm{sec}$.

\section{B. Linear example}

Consider the linear system (7) and the control $u=K x$ studied in [14], [24] with

$$
A=\left[\begin{array}{cc}
0 & 1 \\
0 & -0.1
\end{array}\right], B=\left[\begin{array}{c}
0 \\
-0.1
\end{array}\right], K=\left[\begin{array}{l}
3.75 \\
11.5
\end{array}\right]^{T} .
$$

Several robust stability conditions dedicated to the previous example of sampled-data systems can be found [6], [8], [15], [19]. In these articles, the main idea is to provided the largest upper-bound $T$ so that the closed-loop system is stable for any asynchronous samplings whose period is lying in $[0, T]$. It was shown in [19] that the system remains stable with the upper-bound $T=1.729$.

To provide an efficient event-triggered algorithm, the proposed Lyapunov matrix is taken from [19] with $T=0.2$ and

$$
P=\left[\begin{array}{ll}
21.213 & 10.843 \\
10.843 & 20.666
\end{array}\right], \quad \alpha=0.17 .
$$

Figure 3 shows the simulations results of the closed system using the continuous-time controller, and the event-driven Propositions 2 and 3 with $\bar{\alpha}=10^{-3}$ and $\lambda=0.15$. The first and the second algorithms require respectively 12 and 10 sampling instants over a simulation time of $20 \mathrm{sec}$. 

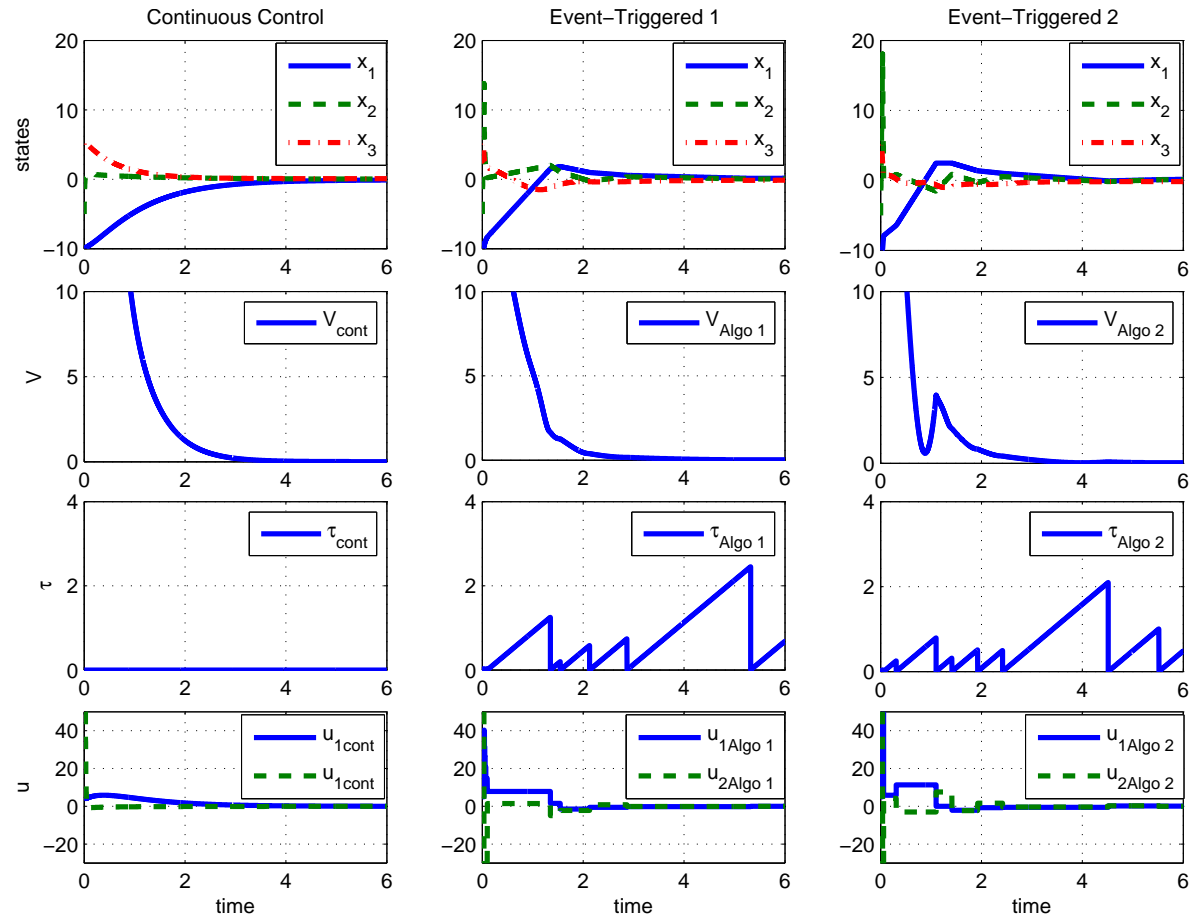

Fig. 2. Simulation of the nonlinear system (11) with the controller (12) in continuous-time (on the left) and in the sampled case following the eventtriggered algorithms given by Theorems 1 and 2 (respectively on the middle and on the right). From the top to down: time-evolution of the states, of the $V$-function, of the $\tau$-variables and of the control values.

Using Proposition 4, the algorithm derived from Proposition 2 leads to the following bounds on difference between two successive sampling instants $T_{\min }^{1}=0.978$ and $T_{\max }^{1}=$ 6.96. Similarly, the algorithm derived from Proposition 3 leads to $T_{\min }^{2}=1.02$ and $T_{\max }^{2}=14.91$. Using both event-triggered algorithms, it is possible to use samplings whose length between two successive sampling instants could be greater than the upper-bound obtained using robust approaches from [6], [8], [15], [19]. This shows the main interest of the proposed method.

\section{CONCLUSIONS}

In this paper using a Lyapunov-like function, two algorithms for the design of event-triggered algorithm are designed. It is assumed that a stabilizing controller for the continuous control system is given. Both event-triggered algorithms need to consider a closed-loop system with a mixed discrete/continuous dynamics (namely this is a hybrid system). Some numerical simulations illustrate the stability properties of both algorithms.

In a forecoming work, the performance issue should be analyzed. It is remarked that the event-triggered algorithms have a different performance. The first one seems to ensure a good speed of convergence on numerical simulations, whereas the second event-triggered algorithm allows less jumps and thus needs to compute less often the control variables. The advantages and disadvantages of each algorithm will be studied more precisely in a forecoming work, for a theoretical point of view (e.g. by estimating a priori the number of switches), or on applications (to understand which algorithm is better depending on the application).

\section{REFERENCES}

[1] A. Anta and P. Tabuada. To sample or not to sample: self-triggered control for nonlinear systems. IEEE Transactions on Automatic Control, 55(9):2030-2042, 2010.

[2] K.J. Ȧström and B.M. Bernhardsson. Comparison of Riemann and Lebesgue sampling for first order stochastic systems. In Proceedings of the $41^{\text {st }}$ IEEE Conference on Decision and Control, volume 2, pages 2011-2016, Las Vegas, Nevada, USA, 2002.

[3] C. I. Byrnes and A. Isidori. New results and examples in nonlinear feedback stabilization. Systems \& Control Letters, 12:437-442, 1989.

[4] T. Chen and B.A. Francis. Optimal sampled-data control systems. Springer-Verlag, Berlin, Germany, 1995.

[5] R.A. Freeman and P. Kokotović. Robust nonlinear control design. State-space and Lyapunov techniques. Birkhäuser, 2008.

[6] E. Fridman. A refined input delay approach to sampled-data control. Automatica, 46(2):421-427, 2010.

[7] E. Fridman, A. Seuret, and J.-P. Richard. Robust sampled-data stabilization of linear systems: An input delay approach. Automatica, 40(8):1141-1446, 2004.

[8] H. Fujioka. Stability analysis of systems with aperiodic sample-andhold devices. Automatica, 45(3):771-775, 2009.

[9] R. Goebel, R. Sanfelice, and A.R.Teel. Hybrid dynamical systems. IEEE Control Systems Magazine, 29(2):28-93, 2009.

[10] W. Heemels, J. Sandee, and P. van den Bosch. Analysis of eventdriven controller for linear systems. International Journal of Control, 81(4):571-590, 2009

[11] J.P. Hespanha, P. Naghshtabrizi, and Y. Xu. A survey of recent results in networked control systems. Proceedings of the IEEE, 95(1):138$162,2007$.

[12] H.K. Khalil. Nonlinear Systems. Prentice-Hall, 3rd edition, 2002.

[13] P.V. Kokotovic. The joy of feedback: nonlinear and adaptative. IEEE Control Systems Magazine, 12(3):7-17, June 1992. 

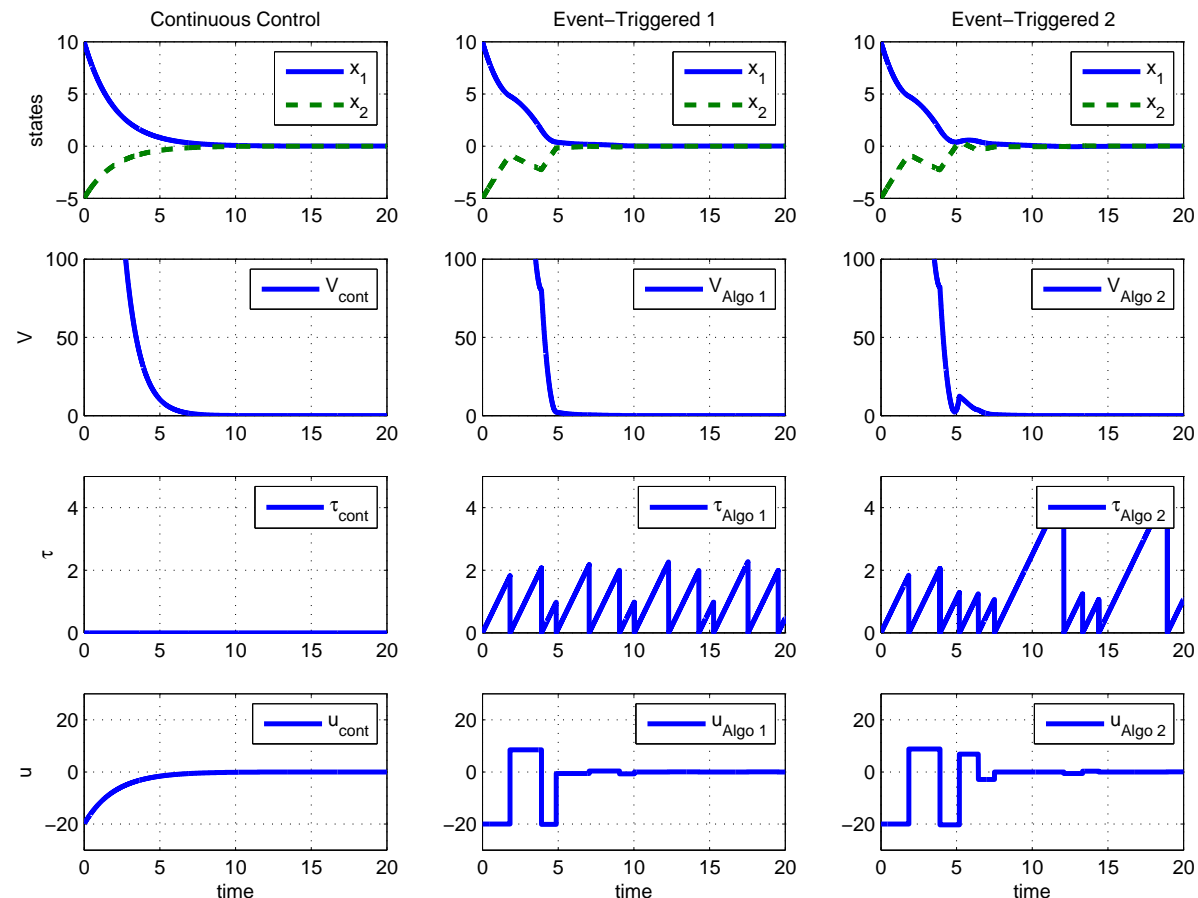

Fig. 3. Simulation of the linear system (7) and the controller $u=K x$ defined in (13) using the continuous-time controller (on the left) and the eventtriggered algorithms from Propositions 1 and 2 (respectively on the middle and on the right). From the top to down: time-evolution of the states, of the $V$-function, of the $\tau$-variables and of the control values.

[14] P. Naghshtabrizi, J.P. Hespanha, and A.R. Teel. Exponential stability of impulsive systems with application to uncertain sampled-data systems. Systems and Control Letters, 57(5):378-385, 2008.

[15] Y. Oishi and H. Fujioka. Stability and stabilization of aperiodic sampled-data control systems: An approach using robust linear matrix inequalities. In Joint $48^{\text {th }}$ IEEE Conference on Decision and Control and $28^{\text {th }}$ Chinese Control Conference, pages 8142-8147, Shanghai, China, 2009

[16] C. Prieur, R. Goebel, and A.R. Teel. Hybrid feedback control and robust stabilization of nonlinear systems. IEEE Transactions on Automatic Control, 52(11):2103-2117, 2007.

[17] C. Prieur, S. Tarbouriech, and L. Zaccarian. Guaranteed stability for nonlinear systems by means of a hybrid loop. In IFAC Symposium on Nonlinear Control Systems (NOLCOS), pages 72-77, Bologna, Italy, September 2010.

[18] J. Sandee, W. Heemels, and P. van den Bosch. Event-driven control as an opportunity in the multidisciplinary development of embedded controller. In IEEE American Control Conference, pages 1776-1781, Portland, Oregon, USA, 2005.

[19] A. Seuret. Stability analysis for sampled-data systems with a timevarying period. In Joint $48^{\text {th }}$ IEEE Conference on Decision and Control and $28^{\text {th }}$ Chinese Control Conference, pages 8130-8135, Shangai, China, December 2009.

[20] A. Seuret. A novel stability analysis of sampled-data systems with applications to multi-rate sampling and packet loss. Provisionnally accepted to Automatica, 2011.

[21] E.D. Sontag. Input to state stability: Basic concepts and results. In Nonlinear and Optimal Control Theory, pages 163-220. SpringerVerlag, Berlin, 2007

[22] M. Velasco, P. Martí, and E. Bini. On Lyapunov sampling for eventdriven controllers. In Joint $48^{\text {th }}$ IEEE Conference on Decision and Control and $28^{t h}$ Chinese Control Conference, pages 6238-6243, Shanghai, China, 2009.

[23] S. Zampieri. A survey of recent results in Networked Control Systems. In Proc. of the $17^{\text {th }}$ IFAC World Congress, pages 2886-2894, Seoul, Korea, July 2008.
[24] W. Zhang, M.S. Branicky, and S.M. Phillips. Stability of networked control systems. IEEE Control Systems Magazine, 21(1):84-99, 2001. 